\title{
Hyper sensitive plasmonic optical system for pollutant detection
}

\author{
Nicolas Javahiraly $^{(1)}$, Hamza Settouti ${ }^{(1)}$, Nacer Boubiche ${ }^{(1)}$ and François Le-Normand ${ }^{(1)}$. \\ ${ }^{(1)}$ Université de Strasbourg, Laboratoire ICube UMR 7357 \\ 23, rue du Loess - 67037 Strasbourg \\ FRANCE \\ Contact: n.javahiraly@unistra.fr
}

\begin{abstract}
The detection of micro pollutants by new innovative systems is one of the important issues of our society. This study is dedicated to innovative pollutant sensors exploiting the interaction properties between light and original nanostructured materials, in order to create a real jump in performance in terms of detection limit, quantification and sensitivity. The detection of our pesticide is based on the variation of the optical properties of the materials used in the presence of the molecule to be detected. We propose two ways of investigation that are (i) the Surface Plasmon Resonance detection (SPR) in Kretschmann configuration and (2) the use of an original functionalized nano structured organization based on the use of functionalized gold nanoparticles.
\end{abstract}

Keywords: Pesticide detection, plasmonics, metal hybrid.

\section{INTRODUCTION}

Glyphosate is the most widely used pesticide in the world, about 650000 tonnes used in 2011. It is a systemic total foliar herbicide, i.e. non-selective, absorbed by the leaves and at generalized action. It has been much talked about in the media in recent years on the issue of renewal of its authorization, or not, given that it is the subject of an authorization from placing on the market valid until 31 December 2015. The toxicity of glyphosates was measured on fauna, flora and the environment. It was ranked among five other herbicides in March 2015 as "possible" or "probable" carcinogens by the World Health Organization. The most commonly used techniques for the detection of glyphosate are usually heavy and very expensive techniques such as tandem mass spectrometry (ISO 16308: 2014) or fluorescence spectroscopy (ISO 21458: 2008).

The goal of this work is to develop and optimize a fast, simple and inexpensive sensor for the detection of glyphosate and if possible on site. Our choice fell on the technique SPR (Surface Plasmon Resonance) which is a simple and effective optical detection technique. The literature review allowed us to confirm that optical methods are more efficient and reach detection limits that exceed the other methods.

The State of Art shows several useful and efficient techniques such as electrochemical detection of glyphosate using peroxidase horseradish immobilized on a sulfonated polymer matrix [1], where the detection limit obtained by this method is $1.70 \mu \mathrm{g} / \mathrm{L}$, which therefore provides sufficient sensitivity for the detection of glyphosate in real samples.

The use of nanoparticles (NP) can be achieved too by a spectroscopic detection of the pollutant in water with silver nanoparticles obtained by laser ablation [2].

Silver nanoparticles, produced by laser ablation in a liquid medium (PLAL), in a citrate-water solution were used as a substrate for the detection of glyphosate in water. The PLAL parameters and the proportion of citrate used as a stabilizing agent were chosen to form a colloidal solution which remains stable during the measurement period when glyphosate is added to the colloid. Two principles of transduction were studied: colorimetric, using the UV-Vis extinction spectrum of the LSPR depending on the concentration of analyte, and SERS carrying not only on the concentration of the analyte but also on the interaction between the analyte and the colloidal silver substrate. The detection limits of $0.9 \mathrm{mg} / \mathrm{l}$ and $3.2 \mathrm{mg} / \mathrm{l}$ were obtained with UV-VIS and SERS (Surface Enhanced Raman 
spectroscopies) respectively. For this approach, colorimetric transduction using the ratio between extinction bands and also transduction with higher specificity using SERS bands are shown. Tests with interferents and glyphosate indicate that the UV-Vis and SERS spectra have glyphosate-dependent characteristics.

Another way to detect and to quantify the pollutant detection is the fiber optic sensor way [3]: in the system, the authors chose an optical fiber coated with a layer of gold nanoparticles and functionalized with cysteamine to detect glyphosate in water. The operation of the sensor is based on the reaction between the glyphosate molecules and the cysteamine which modifies the effective refractive index of the optical cladding.

In the presence of glyphosate-containing water, spectral changes occur in the attenuation bands of the long-period fiber grating, thus allowing interrogation of the sensor at both wavelength and intensity.

The sensor relies on the high sensitivity of long-lived fiber gratings that operate in the visible spectral range near the turning point, as well as on the resonance of plasmons between the cladding and the gold nanoparticles. The resonance between LPG and LSPR has been shown to increase the sensitivity of LPG attenuation bands to glyphosate in the sample.

The sensor configuration proposed in this study was tested with water samples contaminated with glyphosate with a detection limit of approximately $0.02 \mu \mathrm{M}$. Functionalization of gold nanoparticles deposited on LPG with cysteamine adds selectivity to the traditional LPG refractometric sensor. LPG operating in the visible spectral range, when coated with gold nanoparticles functionalized with cysteamine, can not only detect glyphosate but also quantify the concentration of glyphosate in water samples.

The analysis of the state of the art has shown us that the distribution is mainly focused on spectrometric measurement techniques: mass spectrometry, fluorescence spectrometry and UV-Vis spectrometry, the most used techniques in this field. From the figure 1, it is clear that optical detection seems to be a viable alternative technique reaching detection limits in the order of $\mu \mathrm{g} \cdot \mathrm{l}^{-1}$.

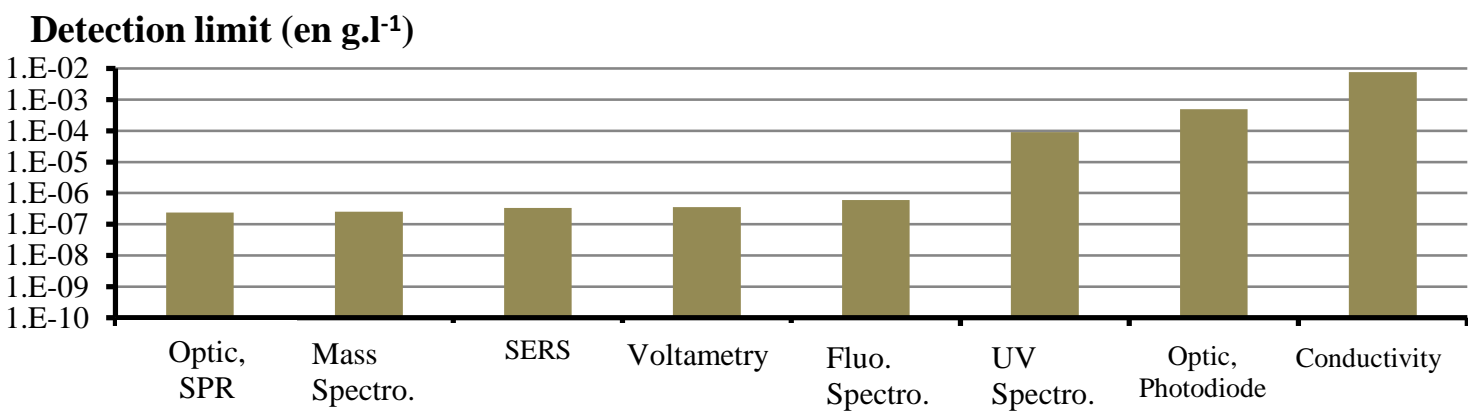

Figure 1: Comparison of the detection limit of different glyphosate detection techniques.

This is the order of magnitude corresponding to the environmental quality standards of pesticides [4]. Moreover, as we have seen, optical techniques offer more innovative perspectives in terms of studies of new materials, cost, on-site detection possibilities, etc.

\section{SENSOR PRINCIPLE: SURFACE PLASMON RESONANCE}

Surface plasmon is a physical phenomenon that occurs at the interface between a dielectric and a metal following the interaction of an electromagnetic wave with electrons free of this interface. This phenomenon is described by the oscillation of electrons, their propagation gives rise to an evanescent electric field whose characteristics are related to the permittivity $(\varepsilon)$ of the metal and the dielectric. The interface then acts as a waveguide for the electromagnetic wave.

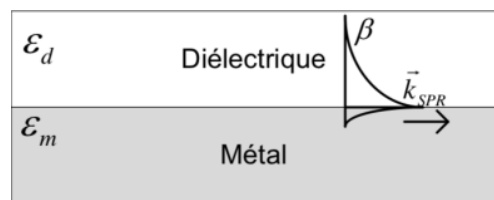

Figure 2: Representation of the evanescent SPR wave at the metal / dielectric interface. 
The propagation of the evanescent wave at the dielectric/metal interface can be described by the electromagnetic theory and thus explained by the resolution of the Maxwell equation at the interface. The corresponding electric field varies periodically in the plane of the surface and exponentially decreases in the directions perpendicular to this plane. The evanescent wave at the surface is associated with the presence of surface charges, due to the discontinuity of the normal component of the electric field oscillating perpendicularly to the surface.

It is therefore a transverse electromagnetic wave defined by [5]:

$$
\beta=\frac{\omega}{c} \sqrt{\frac{\varepsilon_{d} \varepsilon_{m}}{\varepsilon_{d+} \varepsilon_{m}}}=k \sqrt{\frac{\varepsilon_{d} \varepsilon_{m}}{\varepsilon_{d+} \varepsilon_{m}}}
$$

where $c$ is the speed of light in a vacuum, $\omega$ is the angular frequency, $\varepsilon_{d}$ and $\varepsilon_{m}$ are respectively the permittivity of the dielectric and of the metal and $\mathrm{k}$ represents the wave number.

A metal is generally absorbent for an electromagnetic wave. This results in a complex permittivity $\left(\varepsilon_{\mathrm{m}}=\varepsilon_{\mathrm{m}}^{\prime}+i \varepsilon_{\mathrm{m}}^{\prime}\right)$. In this case, equation (1) has a real part and an imaginary part $\left(\beta=\beta^{\prime}+i \beta^{\prime \prime}\right)$. The real part $\beta^{\prime}$ represents the propagation mode at the interface and the imaginary part $\beta$ " represents the attenuation.

The attenuation can be related to the propagation length of the wave. Considering an attenuation of a factor 1/e of the energy of the wave, then it is possible to describe the length of L propagation by [5]:

$$
L=\frac{1}{2 \beta^{\prime \prime}}
$$

There are several methods of coupling to obtain an excitation of the surface plasmons, in our study we chose the case of prism coupling with Kretschmann configuration and gold as metal.

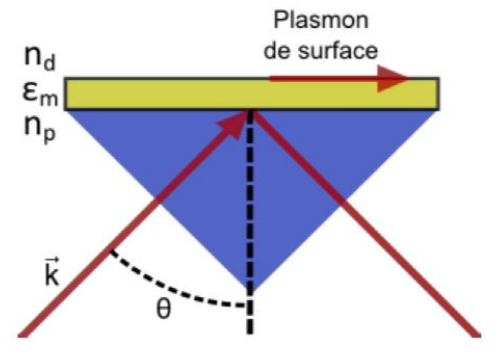

Figure 3: Photon-plasmon coupling method by prism in the Kretschmann configuration [6].

In the case of prism coupling, the tangential component of the wave vector of the incident light is increased by the prism, thanks to its refractive index $\left(n_{p}=\sqrt{\varepsilon_{p}}\right)$ which is larger than that of the air.

To excite the surface plasmons, the incident light must propagate in the refractive index prism $n_{\mathrm{p}}$ with an angle $\theta$ greater than the critical angle of total reflection. It is thus reflected by the gold surface, which is deposited prism surface. An evanescent wave is then created and propagates in the metal. If the thickness of the metal layer is sufficiently thin (less than $100 \mathrm{~nm}$ ), the evanescent wave penetrates sufficiently to couple with the surface plasmons. Under the right angle and wavelength conditions, the surface plasmons are excited at the interface between the thin gold layer and the dielectric medium and the intensity of the reflected light decreases tremendously since a portion of the energy of the evanescent wave is absorbed by the surface plasmons. There is a SPR pic creation at the resonant wavelength. The gold surface is then functionalized to "catch" the pollutant. When the pollutant is detected, there an SPR shift that occurs. By measuring this SPR pic shift, we can determine the pollutant concentration very efficiently. 


\section{EXPERIMENTAL PART AND SIMULATION}

\subsection{Experimental Set Up:}

We compared the different uses of the SPR technique to determine the technique we will use. We had the ability to detect a wide range of wavelengths. We therefore turned to a fixed-angle and variable-wavelength SPR configuration. The following figure presents the experimental set up: The source is a halogen lamp emitting from 360 to $1100 \mathrm{~nm}$.

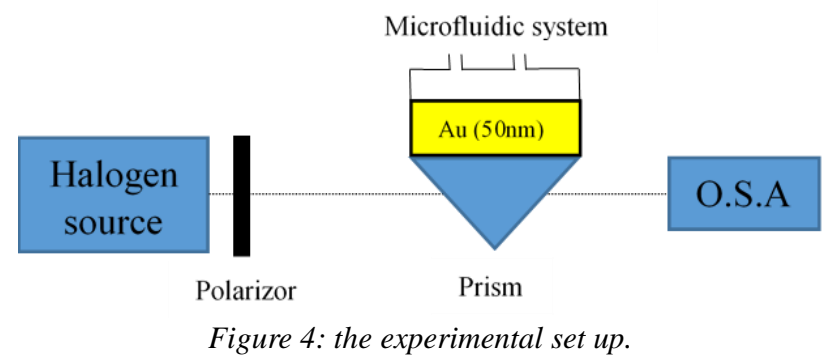

The Optical Spectrum Analyzer permits to collect the output signal and the prism is a BK7 prism. A microfluidic cell has been design and achieved by a 3D printing system and permit to bring the pollutant in water solution at different concentrations.

The deposition of the gold layers was carried out by an electron beam evaporation method. The thickness of the deposits is studied by the FDTD simulation to determine the optimal conditions of generation of the plasmonic waves. The interest of the microfluidic cell, made by 3D printing, lies in the possibility of successively depositing different solutions on the surface of the prism, without having to move or clean it. At the same time, it enables real-time measurements and constant solution injection rates, facilitating biodetection by SPR technique.

Firstly, the validity of the SPR assembly will be tested with water and different glucose solutions prepared in concentrations of the order of mol.1 ${ }^{-1}$, to verify that the modification of the refractive index is observable via the movement of the SPR peak. Finally, the editing will be used to try to detect the molecule of glyphosate by an indirect method.

\subsection{FDTD (Finite Difference Time Domain) simulations}

The method chosen for the simulations is the FDTD (Finite Difference Time Domain), a method of temporal resolution of Maxwell's equations [7-8]. The method uses finite differences to approximate differential equations. The discretization of the components of space then allows a temporal resolution of the equations of the light propagation field. Plasmons, by definition, are oscillations of plasma that can be described by Maxwell's equations, which makes it possible to use FDTD simulation.

The FDTD method consists in transforming the partial differential equations that we want to solve into finite difference equations. This is done using Taylor's limited serial developments. Let us assume that $\mathrm{f}(\mathrm{h})$ is a continuous and derivable function representing a component of the electric or magnetic field.

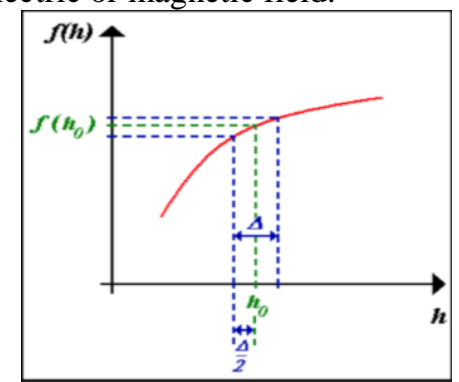

Figure 5: Representation of the principle of calculation of the derivative first of $f(h)$ in ho approximated by a limited series development of Taylor to the right and left of ho [9].

It is possible to obtain a centered approximation of the first derivative of $\mathrm{f}(\mathrm{h})$ in $h_{0}$ by a limited Taylor series development to the right and left of the point $h_{0}$ : 
- Approximation on the right :

$f\left(h_{0}+\frac{\Delta}{2}\right)=f\left(h_{0}\right)+\frac{\Delta}{2} f^{\prime}(h) l_{h=h_{0}}+\frac{1}{2 !}\left(\frac{\Delta}{2}\right)^{2} f^{\prime \prime}(h) l_{h=h_{0}}$

- Approximation on the left :

$f\left(h_{0}-\frac{\Delta}{2}\right)=f\left(h_{0}\right)-\frac{\Delta}{2} f^{\prime}(h) l_{h=h_{0}}+\frac{1}{2 !}\left(\frac{\Delta}{2}\right)^{2} f^{\prime \prime}(h) l_{h=h_{0}}$

by subtracting these two equations and dividing by $\Delta$, we obtain:

$$
\left.\frac{\partial f}{\partial h}\right\rfloor_{h=h_{0}}=\frac{f\left(h_{0}+\frac{\Delta}{2}\right)-f\left(h_{0}-\frac{\Delta}{2}\right)}{\Delta}+o(\Delta)^{2}
$$

where $o(\Delta)^{2}$ represents the second order error that is made and ignored.

The FDTD method is based on the double discretization, both temporal and spatial. The entire structure to be analyzed is then discretized according to a uniform grid of steps $\Delta x=\Delta y=\Delta z$ for 3D space and time with a time increment $\Delta t$. Thus, each partial derivative (spatial or temporal) in the evolution equations of the electric and magnetic field components will be replaced by a second-order Taylor development.

\section{RESULTS}

\subsection{Simulations results}

\subsubsection{Influence of the thickness of the gold layer}

The need to deposit a layer of gold on the basis of the prism requires determining which thickness is optimal to generate a plasmon sufficiently effective for it to be usable (before functionalization).

For different thicknesses of the gold layer, a different behavior of the light reflected on the gold surface is observed (Figure 6).

a) Gold layer thickness $=25 \mathrm{~nm}$

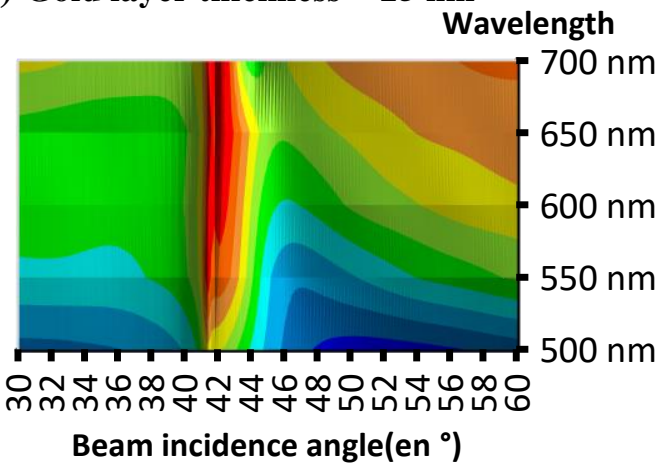

b) Gold layer thickness $=\mathbf{5 0} \mathrm{nm}$

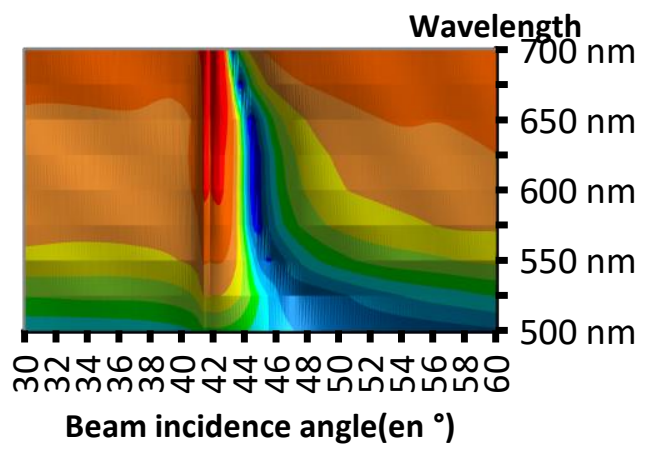




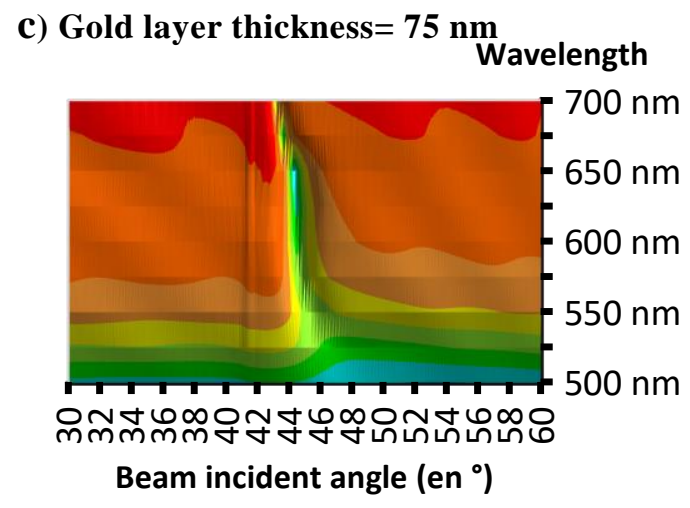

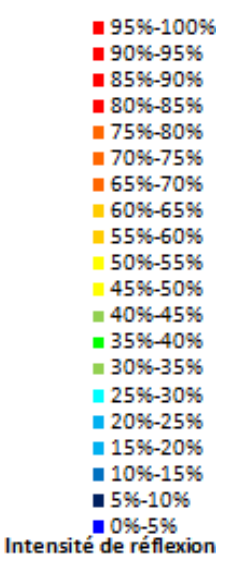

Figure 6: 3D SPR profile of the intensity of reflected light as a function of wavelength and angle of incidence. Influence of the thickness of the gold layer on the prism.

If the thickness of the gold layer is too thin (6a), generally less than $40 \mathrm{~nm}$ thick, a peak of reflectivity is observed at an angle of incidence of $42^{\circ}$ opposite to the phenomenon we want to see, i.e. the SPR peak symbolized by a drop in intensity [10].

If the thickness of the gold layer is too thick (6c), a 60\% drop in intensity is observed for the light reflected at an angle of $45^{\circ}$. The SPR phenomenon is visible but weakly marked.

When the thickness of the gold layer is optimal $(50 \mathrm{~nm})$, an SPR peak is observed at $580 \mathrm{~nm}$ for an angle of $45^{\circ}$. The intensity drop is more than $90 \%$, the SPR peak is well resolved but can only be observed by scanning the incidence angle (figure 7).
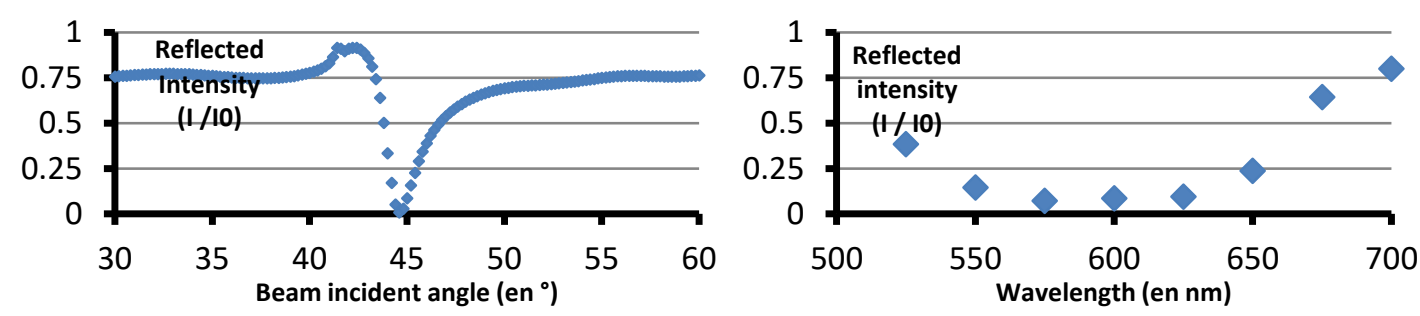

Figure 7: 2D SPR profile for a gold thickness of $50 \mathrm{~nm}$. Left at a fixed wavelength of $580 \mathrm{~nm}$, right at a fixed angle of $45^{\circ}$.

\subsubsection{System optimization}

The deposition of a gold layer on glass is complicated in practice. This is due to the low adhesion between the two materials. The gold layer is therefore generally preceded by a layer of another metal (e.g. chromium, titanium) to obtain a stable substrate. A thin layer of Chromium $(5 \mathrm{~nm})$ was deposited on the prism to fix the gold. The following figure (figure 8) shows the intensity obtained as a function of wavelength. 


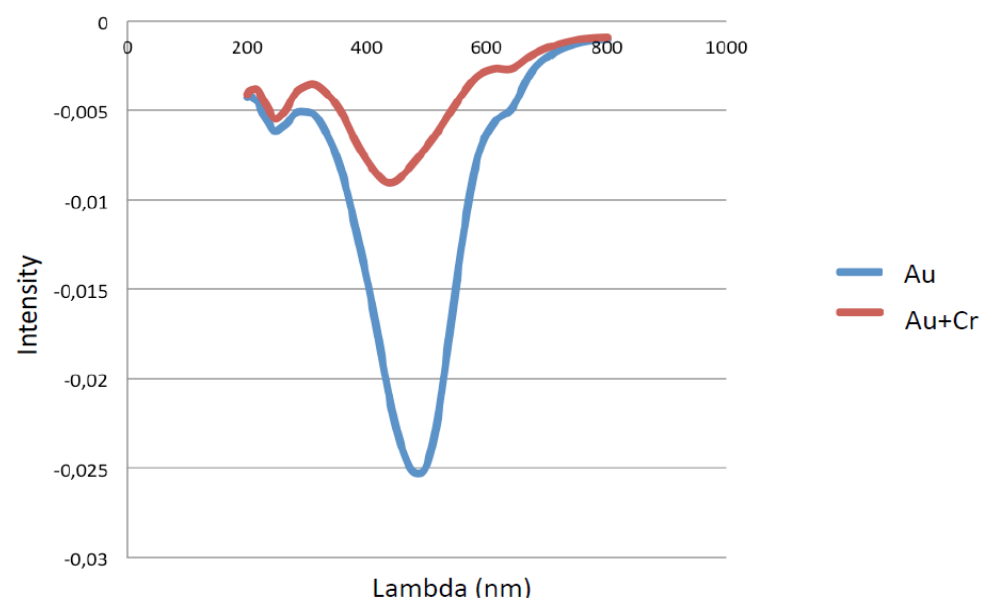

Figure 8: Transmission of gold and gold with chromium as a function of wavelengths.

The deposition of the chromium layer on the prism before the gold resulted in a decrease in amplitude and a shift of $50.748 \mathrm{~nm}$.

\subsubsection{Pollutant detection}

In this simulation we added water to the gold, we obtained as a result the red curve (figure 9), then glyphosate was added to the water (the purple curve) with a refractive index $n=1.576$ to $100 \%$.

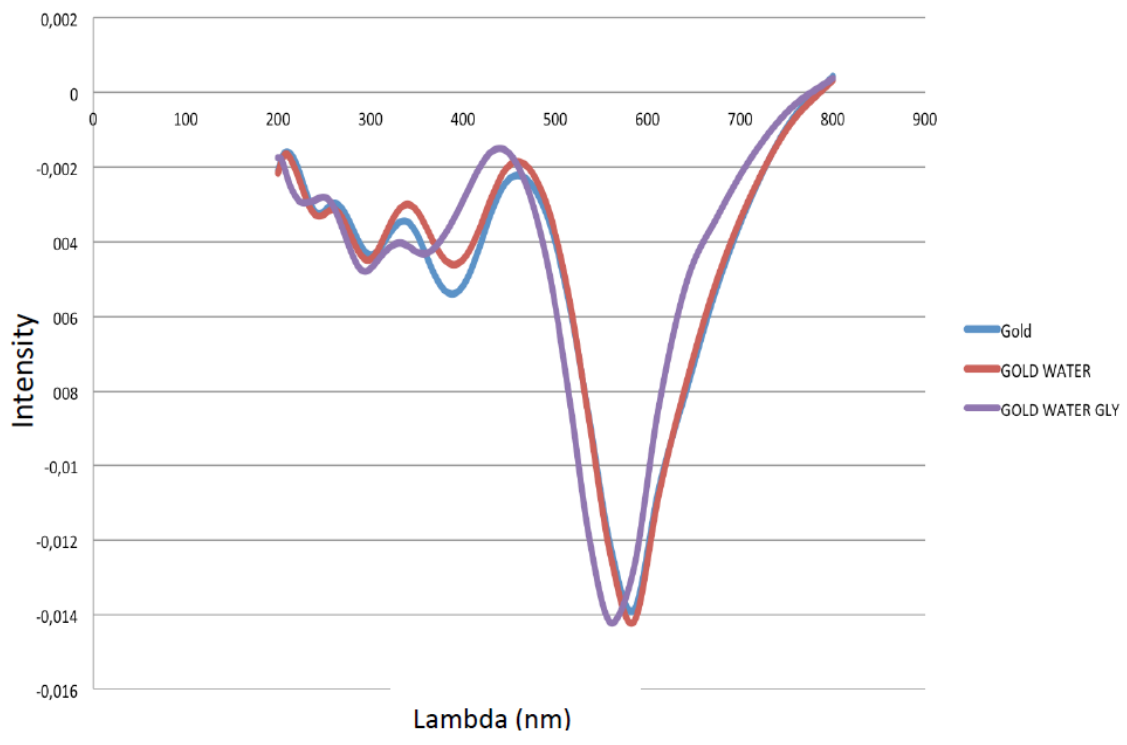

Figure 9: Transmission of gold, gold with water and gold with polluted water according to wavelengths.

After interposing the curves on the same graph, we notice a shift to the left of the SPR peak of about $20 \mathrm{~nm}$, which indicates that glyphosate has been detected. 


\subsection{Experimental results}

\subsubsection{Glucose-using test}

After optimizing our experiment set up with water, we tested different glucose solutions to see if the shift SPR was visible for solutions with a different refractive index. The tests were carried out with different substrates prepared under the same conditions (glass slide / chrome / gold).

The following table presents the refractive index (measured by a refractometer) and SPR pic shift as a function of glucose concentrations.

\begin{tabular}{|c|c|c|}
\hline $\begin{array}{c}\text { Glucose } \\
\text { concentration }(\mathrm{M})\end{array}$ & $\begin{array}{c}\text { Refractive } \\
\text { index }\end{array}$ & $\begin{array}{c}\text { SPR pic } \\
\text { shift (nm) }\end{array}$ \\
\hline 0 & 1.3310 & 0 \\
\hline 0.25 & 1.3314 & 1.277 \\
\hline 0.50 & 1.3320 & 2.298 \\
\hline 0.75 & 1.3327 & 3.064 \\
\hline 1 & 1.3334 & 4.086 \\
\hline
\end{tabular}

Table 1: Refractive index and SPR pic shift as a function of glucose concentrations

We note that the displacement of the SPR pic is linear as a function of the refractive index of the solution, and the glucose concentration.

For a glucose concentration from 0 to $1 \mathrm{M}$, we measure SPR peak shift from 0 to $4 \mathrm{~nm}$, the refractive index increases linearly from 1.3310 to 1.3334 .

This linear dependence demonstrated by the measurement can be used in a calibration process if we want to detect the presence of glucose in an unknown solution.

\subsubsection{Perspectives and next step}

Following the previous study and the results obtained with glucose, the next two steps are the chemical functionalization to the gold layer and the synthesis of gold nanoparticles that can be used too to detect the pollutant.

The Figure 10 shows gold nanoparticles produced by molecular jet epitaxy (MBE) in the experimental phase on different substrates deposited on glass.

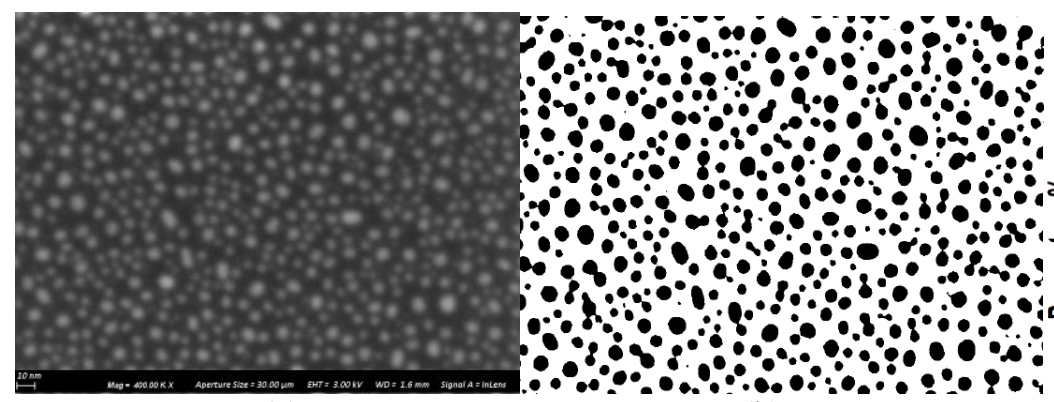

(a) (b)

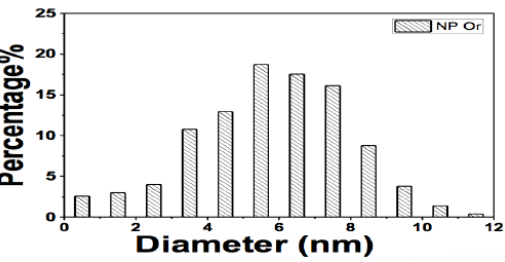

(c)

Figure 10: (a) Gold nanoparticle image by scanning electron microscope; (b) Processed image of gold nanoparticles; (c) Distribution of gold nanoparticles according to diameter.

Figures 10 (a) and 10 (b) show the results obtained after optimal deposition of nanoparticles of gold on a $15 \mathrm{~nm}$ thick substrate layer developed in the laboratory. The observation of the optimal SEM sample and its treatment shows NPs with an average diameter of $6 \mathrm{~nm}$ (figure $10 \mathrm{c}$ ) with an average inter NP distance of $5 \mathrm{~nm}$. The image obtained by SEM is blurred due to sample contamination during focusing of the scanning electron microscope. 


\section{CONCLUSION}

The objective of this work was to develop and optimize a recognized optical technique, called SPR surface plasmon resonance, which is applied for the dynamic study of ligand-receptor interactions.

In order to demonstrate the feasibility of such a system applied to the detection of pollutants, a rigorous simulation study has made it possible to develop an FDTD code to observe the behavior of the SPR peak under different conditions.

The current work in progress has enabled us to set up the experimental set-up showing an SPR peak obtained with a 50 $\mathrm{nm}$ thick solid gold layer. The experimental set up has been validated by different tests using glucose solutions at different concentrations. The next step is the chemical functionalization of the gold layer and gold nanoparticles.

\section{ACKNOWLEDGMENTS}

The authors would like to thank the students who contributed to this study, ICube Institute and the InCA transversal axis of the ICube institute.

\section{REFERENCES}

[1] Songa, E. A., Arotiba, O. A., Owino, J. H., Jahed, N., Baker, P. G. and Iwuoha, E. I., "Electrochemical detection of glyphosate herbicide using horseradish peroxidase immobilized on sulfonated polymer matrix," Bioelectrochemistry. 75(2), 117-23 (2009).

[2] De Góes, R. E., Muller, M., Fabris, J. L., "Spectroscopic Detection of Glyphosate in Water Assisted by LaserAblated Silver Nanoparticles," Sensors. 17(5), 954 (2017).

[3] Heidemann, B. R., Chiamenti, I., Oliveira, M. M., Muller, M. and Fabris, J. L., "Functionalized Long Period Grating-Plasmonic Fiber Sensor Applied to the Detection of Glyphosate in Water," Journal of Lightwave Technology. Vol. $36, n^{\circ} 4,863$ (2018).

[4] INERIS 2014: https://substances.ineris.fr/fr/substance/getDocument/2984

[5] Homola, J. and Dostalek, J., [Surface plasmon resonance base sensors.Techniques], Springer, Verlag Berlin Heidelberg, 251 (2006)

[6] Latendresse V., Report [Développement d'un biocapteur combinant la plasmonique et l'électrochimie] (2013).

[7] Yee, K. S., "Numerical Solution of Initial Boundary Value Problems Involving Maxwell's Equations in Isotropic Media", IEEE Trans. Antennas Propagat., vol. 14, 302-307 (1966).

[8] Taflove A., [Computational electrodynamics:The Finite-Difference Time-Domain Method], Norwood, MA: Artech House Inc. (1995).

[9] Saison-Francioso O., thesis report, [Etude théorique et modélisations par la méthode FDTD de nanostructures plasmoniques : Application à la conception de biocapteurs], Université de Lille (2014).

[10] Ou C., thesis report, [Détection et quantification des polluants], University of Strasbourg (2015). 